\title{
Foilized pouches can prevent the transmission of HIV from mother to child using multi-drug therapies
}

\author{
Robert Malkin
}

Department of Biomedical Engineering, Duke University, Durham, USA

Email: robert.malkin@duke.edu

Received 9 December 2013; revised 9 January 2014; accepted 21 January 2014

Copyright (C) 2014 Robert Malkin. This is an open access article distributed under the Creative Commons Attribution License, which permits unrestricted use, distribution, and reproduction in any medium, provided the original work is properly cited. In accordance of the Creative Commons Attribution License all Copyrights (c) 2014 are reserved for SCIRP and the owner of the intellectual property Robert Malkin. All Copyright (C) 2014 are guarded by law and by SCIRP as a guardian.

\section{ABSTRACT}

Children can become HIV positive (HIV+) from their mother during home birth. If the infant ingests antiretroviral (ARV), medications immediately after birth, the risk of transmission can be dramatically reduced. We have previously proposed the use of foilized, polyethylene-lined pouches to store ARV's. Using the pouch, the mother receives the medication at an antenatal care visit, months before delivery, and if she delivers at home, tears open the pouch and drips the medication into her child's mouth. In this work, we extend the use of the pouch to store a modern ARV, Lamivudine (3TC), often used in multi-drug regimens. Under laboratory conditions, pouches were filled with $3 T C$ and stored at $25^{\circ} \mathrm{C} / 60 \%$ relative humidity (RH) for twelve months. We found that the 3TC was stable throughout the year (maximum $5.6 \%$ of labeled concentration change). The preservatives were somewhat degraded by the act of repackaging the medicine, but sufficient preservatives remained to maintain the medication. The same impurities were identified in the 3TC stored in the pouches and the samples removed from the bottles indicating that the pouches do not introduce new impurities (impurities that are not already introduced by the bottle). We conclude that the pouch can preserve this modern ARV for up to twelve months.

\section{KEYWORDS}

PMTCT; HIV; AIDS; Anti-Retroviral Preservation; Home Birth

\section{INTRODUCTION}

HIV can be transmitted from the mother to child during birth, causing hundreds of thousands of new pediatric
HIV cases each year [1]. Some transmission can be prevented by giving the child antiretroviral (ARV) therapy immediately after birth. Yet, many children go without this medication. Less than $33 \%$ of children born to HIV+ mothers receive prophylactic antiretroviral drugs in resource poor settings [2] and even fewer immediately after birth.

The World Health Organization (WHO) recommends that infants receive ARV's immediately after birth [3]. The infants should receive their first dose within 72 hours of birth to be effective, and preferably within 24 hours. Sadly, many clinics have access to the medications but have no way to provide the medication to mothers who give birth at home. Putting the medication in a cup or syringe destroys the medication before the mother can use it at home.

Waiting for mothers to return to the clinic is also not an adequate solution. For example, 57\% of all Tanzanian women delivered at home in 2005 [4], many of whom did not return to the clinic for weeks or months. Mothers may not return to the clinic because they do not have funds or means for transport, feel too weak to travel or fear stigmatization at explaining why they must go to a clinic after delivering an apparently healthy baby.

We introduced the concept of a foilized polyethylene pouch that can store ARV's for up to a year. If a mother ends up delivering at home, she can tear open the pouch and deliver the medication to her child. We have shown that the pouch is superior to packaging single doses of liquid medication in oral syringes, cups, and other ad hoc solutions [5-8].

However, many of the newer antiretroviral therapy protocols call for Lamivudine (3TC) to be used in multidrug regimens. We have not tested this modern ARV. It is not known if this drug specifically, or its use in a multdrug regimen is compatible with the pouch. In this paper we expand on our previous work by showing that the 
pouch can store 3TC for up to one year.

\section{METHODS}

In our lab, we used $2 \mathrm{ml}$ syringes (Becton, Dickinson and Company) and tapered dispensing tips (TT14-DHUV, Techcon Systems, Garden Grove, CA) to fill 265 premade, foilized pouches (Flex-Pak, Custom Product based on material number 210057) with $1.5 \mathrm{ml}$ of 3TC. The pouches were weighted after filling in our lab.

The pouches and bottles from which the medicine was drawn were sent to Southern Testing and Research Division of Microbac Laboratories, Inc. (Wilson, NC). Samples were stored in walk in stability chambers at $25^{\circ} \mathrm{C} / 60 \%$ relative humidity (RH). Pouches were removed from storage after nine and twelve months. Pouches were weighed prior to stability testing and again when pulled. Three pouches were removed for each measurement. The samples from the three pouches were mixed together to form one chemical sample, then separated again for each measurement type.

Flora and fauna challenges (total microbial aerobic, yeast and mold) were performed following USP61 using USP33-NF28 methodology and tested for the presence of E. Coli (USP62) following USP33-NF28 methodology. In addition to determination of the ARV concentrations, levels of preservatives in the samples were estimated using independently prepared standards following a Microbac-specific protocol (DPHR-966.02) based on the USP Lamivudine API monograph. Finally, the chromatographs were analyzed to determine if impurities were induced in the medication or extracted from the plastic.

\section{RESULTS}

Table 1 shows that the 3TC was stable in the pouches throughout the twelve months. The maximum observed drop in concentration was $5.6 \%$, as opposed to $4.6 \%$ in a bottle stored at 5 degrees centigrade. These values are well with the $10 \%$ allowed by USP.

The pouches prevented nearly all water loss. The average loss in weight was 0.0035 grams. The maximum loss in weight in any pouch over 12 months in any storage condition was 0.0041 gams $(0.27 \%$ of the beginning average weight).

There was a drop in preservative levels in the 3TC, probably due to handling (Table 1). Handling exposes the medicine to mechanical stress, light and oxygen. However, there remained sufficient concentrations of preservatives that the concentration of the active ingredient was stable and the medicine could not support floral or fauna growth.

The same impurities were found in the stored bottle and the stored pouches, indicating that the pouches do not introduce contaminants into the medication. All im-
Table 1. The percent degradation of 3TC solution fell well within the USP 32 NF 27 standard $( \pm 10 \%)$ even after twelve months in the pouch. Preservatives (parabens) act as sacrificial oxidants and their loss may limit shelf life. However, this data shows that despite losses, probably attributable to filling and sealing, the parabens remain in sufficient quantities to preserve the medication.

\begin{tabular}{cccc}
\hline Time & Pouch & Bottle & Bottle \\
\hline Months & $25^{\circ} \mathrm{C} / 60 \%$ RH & $25^{\circ} \mathrm{C} / 60 \% \mathrm{RH}$ & $5^{\circ} \mathrm{C}$ \\
& \multicolumn{3}{c}{ Methyplaraben $(\mathrm{mg} / \mathrm{mL})$} \\
0 & $2.0^{*}$ & $2.0^{*}$ & $2.0^{*}$ \\
9 & 1.3 & 1.4 & 1.4 \\
12 & 1.4 & 1.4 & 1.4 \\
& & Propylparaben (me/mL) & \\
0 & $0.2^{*}$ & $0.2^{*}$ & $0.2^{*}$ \\
9 & 0.1 & 0.2 & 0.2 \\
12 & 0.1 & 0.2 & 0.2 \\
& & $3 T C$ (\% Label Concentration) \\
0 & & $100^{*}$ & $100^{*}$ \\
9 & 94.4 & 99.9 & 95.4 \\
12 & 100.8 & 99.8 & 100.9 \\
\hline
\end{tabular}

*indicates data provided by manufacturer.

purities were recorded at levels well below $1 \%$.

\section{DISCUSSION}

Before our work with the pouch, there was no easy way for mothers who deliver at home to reduce the risk that their children would become HIV+. We have presented the first technique that could provide a reasonable means for mothers to medicate their children immediately after home birth.

When compared to a syringe or cup, the medication is preserved for far longer, up to one year. This allows for packages to be filled and sealed at a central pharmacy and then distributed at a rural clinic or health post well before the mother is to deliver. When a mother does end up delivering at home, the use of the pouch is simple and accurate. Even when provided to mothers after delivery, the pouch presents advantages. Using the pouch, the mother does not need to know how to read or use a syringe, leading to more accurate doses. There is also a reduced risk of wastage, since providing a bottle to a mother introduces the possibility of bottle spillage and contamination.

In our previous work, we showed that the pouch can preserve the two most common medications, NVP and AZT. This paper extends that work by showing that 3TC, the third most common ARV can also be stored in the pouch. Thus, we have expanded our work to show that 
even modern, multi-drug regimens using 3TC are compatible with the pouch.

\section{CONCLUSION}

In conclusion, we have now shown that our foilized polyethylene pouch can store and deliver all of the most common anti-retroviral drugs. These drugs can be prescribed and delivered in the pouch individually or as part of a multi-drug regimen. For the large numbers of women who deliver at home, the pouch is the best option to help achieve the goal of an AIDS-free generation.

\section{ACKNOWLEDGEMENTS}

We would like to thank The Saving Lives at Birth program at the US Agency for International Development for the funding for this research.

\section{REFERENCES}

[1] WHO (2009) PMTCT strategic vision 2010-2015: Preventing mother-to-child transmission of HIV to reach the UNGASS and Millennium Development Goals. WHO, Geneva. http://www.who.int/hiv/pub/mtct/strategic_vision.pdf

[2] WHO, UNAIDS, UNICEF (2009) Towards universal access: scaling up priority HIV/AIDS interventions in the health sector. Progress report 2009. WHO, Geneva, 112. http://www.who.int/entity/hiv/pub/tuapr_2009_en.pdf

[3] WHO (2009) Rapid advice: Antiretroviral therapy for HIV infection in adults and adolescents. WHO, Geneva. http://www.who.int/hiv/pub/arv/rapid_advice_art.pdf

[4] UNFPA (2007) The Prime Minister of Tanzania calls on regional leaders to increase health spending to 15 per cent of national budgets and to strive for achieving health MDGs. Press release. http://www.unfpa.org/public/News/pid/308

[5] Berman, A. and Brooke, S. (2006) Sourcing guide: The Nevirapine infant-dose pouch for use in prevention of mother-to-child transmission of HIV/AIDS programs. Version 1. PATH, Seattle, 2. http://www.path.org/publications/files/TS_update_pkg_n vp.pdf

[6] FDA (1999) Guidance for industry: Container closure systems for packaging human drugs and biologic. Section III.F.1.

[7] Malkin, R.A. and Howard, C. (2012) A foilized polyethylene pouch for the prevention of transmission of HIV from mother to child. The Open Biomedical Engineering Journal, 6, 92-97.

[8] Malkin, R.A. (2013) A pouch may be prevent the transmission of HIV from mother to child. Journal of Biomedical Engineering Research, 2, 66-70. 